risent la végétation des moisissures ; la décomposition de la caséine est profonde, il est vrai, mais la formation des acides volatils est fortement ou complètement limitée.

“ Le Penicillium Roqueforti donne dans ce eas une odeur typique du fromage de Roquefort, et comme cela n'a pas été observé dans la culture avec la caséine en milieu acide, il faut considérer l'influence symbiotique des bactéries lactiques comme condition prineipale de la naissance de l'odeur si appréciée dans le fromage de Roquefort.

“ En comparant les résultats présents avec ceux qu'on avait obtenus sur la easéine avec l'acide lactique, on voit que le Penicillium Roqueforti prospère le mieux en présence des bactéries lactiques et du lactose. "

A la page 32 , je dis ce qui suit: "Mes expériences effectuées avec la caséine pure et la culture pure du Penicillium Roqueforti ont complété les observations dans le sens que la formation de l'odeur s'effectue même dans la simple caséine en présence des bactéries lactiques, donc même sans la 'graisse du lait et sans l'action de l'Oidium lactis. Il est évident que la graisse du lait contribue à la formation de l'odeur et, en sa présence, on obtient des cultures d'une odeur plus prononcée que celle qu'on obtient dans la caséine pure. 》)

J'ai trouvé qu'il était nécessaire de compléter, par les présentes remarques, les expériences de BARTHEL, intéressantes et préeieuses, ainsi que les déductions qui en ressortent et qui ont une valeur pour moi d'autant plus grande qu'elles confirment mes observations dans toute leur étendue.

\title{
CE QUE DOIT SAVOIR UN BON CONTRÓLEUR LAITIER ET BEURRIER
}

\author{
par A.-M. LEROY \\ Ingénieur agronome, \\ Chef de travaux à l'Institut National Agronomique.
}

- surre -

\section{(f) Ineluence sur la lactation de certaines substances ou de CERTAINS GROUPES DE SUBSTANCES ALIMENTAIRES.}

Le problème des " actions spécifiques " de certaines substances alimentaires sur la mamelle a donné lieu à des controverses souvent passionnées. De nombreux praticiens attachent une importance, peut-être excessive, à quelques fourrages particuliers, dont ils admettent à priori la suprématie sur tous les autres; mais leur avis est loin d'être partagé par tous. Entraînés par l'intérêt de la question, 
des savants ont soumis à la vérification expérimentale l'hypothèse de ces prétendues influences, et, sauf dans quelques cas très rares, dont nous allons d'ailleurs parler, il n'ont pas abouti à des résultats positifs. C'est qu'en effet, entre les principes utiles des aliments et le lait, il existe un très grand nombre d'intermédiaires, tels que les tissus du tube digestif, le foie, le, lait, la lymphe, et le tissu mammaire lui-même. Nous connaissons seulement la porte d'entrée de ces éléments; nous pouvons également les retrouver à leur sortie de la mamelle, après leur transformation en lactose, matières grasses, caséine, etc. Mais le mécanisme extrêmement complexe de cette transformation nous échappe, et dans l'ignorance où nous sommes de la physiologie de la lactation, il ne nous est pas permis d'affirmer, ou de nier la valeur particulière d'un aliment déterminé, tant que le mode d'action de cet aliment n'a pas fait l'objet d'expériences méthodiques, suffisamment précises, suffisamment prolongées, et d'une réalisation difficile, qui ne peuvent être poursuivies que par des laboratoires dotés d'un personnel nombreux et expérimenté.

10 L'influence de l'alimentation perte. - Il est incontestable que le régime du vert modifie dans un sens favorable la séerétion laitière et beurrière des vaches. Nous ne mentionnerons toutefois ici cette influence que pour mémoire, puisque nous l'avons antérieurement étudiée.

$2^{\circ} L^{\prime}$ influence du «mouillage au pentre ». - L'on prétend assez fréquemment que la distribution d'aliments très aqueux, comme les buvées chaudes à base de tourteaux ou de farines ou encore les soupes composées avec des drêches de distillerie ou de brasserie, augmentent la quantité de lait sécrétée, mais diminuent d'une façon corrélative la teneur en matière sèche de ce lait. Certains tribunaux ont admis cette manière de voir, et des producteurs de lait ont été condamnés pour avoir mouillé leur lait " dans le ventre de leur vache. " Les vérifications expérimentales qui ont été tentées n'ont cependant jamais réussi à faire apparaître une modification durable de la composition du lait (1), sous l'influence d'un accroissement systématique du taux d'humidité des fourrages. Envisagées au point de vue scientifique, les condamnations basées sur ce motif paraissent donc profondément injustes, et il est vraiment regrettable de voir une opinion manifestement erronée survivre encore aux démonstrations qui ont été faites de sa fausseté par des savants impartiaux.

Il ne faut pas confondre avee les tentatives de " mouillage au

(1) Voir à ce sujet une très remarquable étude critique du professeur Ch. Poncher : La Polylactie, parue dans les Annales des Falsifications et des Fraudes année 1917, p. 304. 
ventre " le fait de distribuer aux vaches des boissons alimentaires chaudes, pendant les mois d'hiver. Ces buvées ont fréquemment une action favorable sur la quantité du lait sécrétée, mais elles ne nuisent nullement à sa qualité, c'est-à-dire à sa composition chimique. Cette dernière est fonction de l'individualité de la vache, elle est aussi sous la dépendance, dans une certaine mesure, de son système nerveux, mais elle obéit beaucoup moins facilement qu'on ne le pense aux influences d'ordre alimentaire.

Il convient d'être extrêmement prudent avant de conclure à une action élective quelconque sur la mamelle d'un produit déterminé. On trouve dans les anciens traités de zootechnie le résultat d'une expérience déjà ancienne, due à Gautrelet, qui fait apparaître une brusque modification de la teneur du lait en extrait sec et en beurre, après la distribution d'un seau d'eau.

Il est clair, évidemment, que cette expérience, enfantine par sa conception, semble constituer une preuve manifeste en faveur du mouillage au ventre, dont nous venons de faire précédemment le procès. Mais si l'on analyse d'un peu plus près les choses, l'on constate, d'abord, que des détails manquent en ce qui concerne la température de l'eau bue, ainsi que le mode de prélèvement des échantillons de lait soumis à l'analyse. Or, nous savons que le lait d'une même vache ne se ressemble jamais à lui-même, pendant la durée d'une traite.

Peut-être le lait analysé avant l'abreuvement provenait-il de la traite d'un quartier de mamelle entier? Est-on sûr que le lạit du deuxième échantillon ne provenait pas du début de la traite d'un autre quartier? D'autre part, l'eau très froide a fort bien pu provoquer une action nerveuse réflexe, temporaire, nuisible à la décharge de matière grasse du tissus de la glande mammaire dans les canaux excréteurs. Le froid exerce en effet une action défavorable sur la teneur du lait en beurre, et certains matins d'automne, après une nuit fraîche, il est fréquent d'obtenir de vaches habituellement bonnes beurrières des laits à taux butyreux très bas, qui atteignent à grand peine, par exemple, 20 à 25 grammes par litre. L'on peut donc, en toute logique, s'inscrire en faux contre la conclusion de Gautrelet et n'attacher d'importance qu'aux essais de plus longue durée, poursuivis sur un grand nombre d'animaux, afin d'éviter l'influence des écarts individuels. Nous n'avons pas eu d'autre but, en rapportant cette expérience, que de mettre en garde le lecteur contre les conclusions trop hâtives, tirées d'observations superficielles.

L'on peut d'ailleurs expliquer d'une manière rationnelle l'augmentation de production laitière des vaches sous l'influence des 
buvées chaudes. Ces soupes excitent l'appétit; distribuées le matin, au moment où la température est encore froide, elles augmentent le bien-être des animaux, et ceux-ci, réchauffés, secouent leur indolence naturelle, et consomment plus rapidement le reste de leur ration.

Sans ce stimulant bienfaisant, les bonnes laitières ne mangeraient probablement que des quantités d'aliments insuffisantes pour satisfaire à leurs besoins, et l'état de sous-nutrition relative, qui en résulterait, porterait finalement préjudice à leur production de lait et de beurre. Les soupes chaudes permettent done d'obtenir, croyonsnous, un meilleur ajustement de la ration aux besoins réels des vaches.

$3^{\circ}$ Influence de la teneur en matière grasse de la ration. - L'on a songé à modifier la composition chimique du lait, en agissant sur la teneur en matière grasse de la ration. L'on a pu démontrer, en effet, que les graisses des aliments pouvaient se retrouver, presque sans modification, à la sortie de la mamelle, et il était permis de penser qu'une alimentation riche en graisses pouvait avoir une action favorable sur la teneur en beurre des laits recueillis dans ces conditions.

Les vérifications expérimentales, tentées par divers savants (Kühn, Soxhlet, Maercker, Morgen), ayant fourni des résultats contradictoires, le Conseil supérieur de l'agriculture allemande a eu l'idée de confier à Kellner le soin d'organiser des essais de grande envergure, entrepris sur un plan uniforme par dix stations d'expérimentation. Les expériences en question ont duré trois mois et elles ont porté sur 196 vaches laitières. La teneur en graisse des rations distribuées a varié entre 150 et 700 grammes par tête et par jour. La conception de ce vaste programme, ainsi que la manière dont il a été exécuté, donnent une importance singulière aux conclusions du rapport général à la rédaction duquel Kellner collabora. Ces conclusions ont été purement négatives. Soumises à des régimes plus ou moins riches en graisse, les vaches ont fourni des laits fort comparables à eux-mêmes pendant toute la durée des essais, sous le rapport du taux butyreux. Cependant, d'assez grands écarts individuels ont été observés, ce qui montre la nécessité, lorsqu'il s'agit d'expériences de cette sorte, d'opérer sur un grand nombre d'animaux, placés autant que possible dans des conditions différentes.

$4^{\circ}$ Influence de la teneur en protéines de la ration. - L'influence des protéines de la ration sur la lactation a fait l'objet de très beaux travaux, exécutés aux Etats-Unis et en Allemagne. Ceux-ci ont montré que la distribution de fourrages riches en azote exerce une action stimulante sur la production du lait et du beurre. Autrement, 
dit, l'emploi d'une ration qui contient une quantité plus forte de protéines que ne l'indique la règle du minimum, préeédemment exposée ( 0 gr. 6 par kilogramme de poids vif, 60 grammes par litre de lait), permet d'obtenir une augmentation des quantités de lait et de matière grasse ; mais cette augmentation est de faible envergure. Or, la protéine est généralement un élément coûteux, et une ration coûte d'autant plus cher à établir qu'elle est plus riche en azote. En fait, si l'on envisage la question en se plaçant au point de vue de l'agriculteur, il n'est pas économique de distribuer des fourrages trop fortement azotés; le supplément de lait qu'on peut obtenir dans ees conditions risque d'avoir un prix de revient bien supérieur à sa valeur intrinsèque.

Cependant, la question qui nous occupe mérite d'être prise en considération, lorsqu'il s'agit de préparer une bête en vue d'un concours laitier et beurrier. Il sera bon de se souvenir, à ce moment, de l'influence favorable des fourrages riches en protéine, et d'habituer progressivement lès vaches dont on espère de remarquables performances à consommer des rations comportant une notable proportion de ces aliments. Mais il convient d'opérer avee prudence, car il ne faut pas oublier que l'excès d'aliments protéiques favorise la congestion de la mamelle, particulièrement à eraindre chez certains sujets doués à ce point de vue d'une fâcheuse prédisposition.

50 Influence des condiments. - Les condiments agissent favorablement sur la lactation, parce qu'ils stimulent l'appétit des animaux. Cette action est d'autant plus nette que les fourrages grossiers qui constituent la partie volumineuse de la ration sont moins sapides. Avec un mélange d'aliments recueillis dans de bonnes conditions, et dont le goût plaît au bétail, l'influence de l'addition supplémentaire d'un condiment donné est beaucoup moins marqué. Parmi les subsstances condimentaires, il convient de citer le sel marin, le fenouil, l'anis, le thé de foin, la mélasse. Le succès auprès des éleveurs de certains mélanges du commerce, généralement vendus fort cher, tient uniquement aux condiments qui entrent dans leur composition.

Ajoutons qu'une légère fermentation, telle que celle qui résulte du contact des betteraves hachées et de la menue paille, pendant une dizaine d'heures, provoque la formation dans la masse du fourrage de principes odorants, dont l'action apéritive est manifeste.

$6^{\circ}$ Actions spéeifiques déterminées. - Enfin, des expériences danoises, américaines et allemandes ont mis en évidence l'action particulière des principaux fourrages, dont la distribution, à doses modérées, semble modifier tantôt dans un sens favorable, tantột dans un sens défavorable, la sécrétion du lait et du beurre.

Un premier groupe d'aliments, qui comprend le maïs, l'avoine, 
le tourteau de gluten de maîs, provoquent un aceroissement de la ‘ quantité de lait, accompagné d'une légère diminution de la teneur en matière grasse. La quantité totale de beurre obtenue n'est pas sensiblement modifiée.

Un second groupe, au premier plan duquel il faut eiter le tourtean de palmiste, le tourteau de coprah, les drêches de distillerie de maïs et au second plan, parce que leur action spécifique est moins nette, le tourteau de lin, le tourteau de coton, et les légumes (betteraves, rutabagas, etc.), ne semblent pas modifier la quantité de lait, mais augmentent légèrement la teneur en matière grasse. Ce groupe d'aliments paraît done exercer une influence favorable sur la production du beurre.

Pour donner une idée de l'ordre de grandeur de cette influence, il nous suffira de citer les résultats des expériences entreprises sur les effets du tourteau de palmiste, par les stations expérimentales allemandes, sous la direction de Kellner. D'aprés la moyenne des observations faites sur 200 vaches, ce tourteau augmente le pourcentage de matière grasse de $0,24 \%$. Pour une production moyenne de 10 litres par jour, le supplément de beurre obtenu par l'alimentation au palmiste est de 24 grammes. Ce résultat est done presque négligeable, et il convient de ne pas exagérer l'importanee pratique des actions spécifiques en question.

Dans le troisième et dernier groupe se rangent le tourteau d'œillette, le tourteau de eameline, les déchets de rizerie et, à un moindre degré, le tourteau de sésame. Ces produits ne paraissent pas agir notablement sur la quantité de lait, mais ils diminuent très légèrement le taux butyreux; cet abaissement peut atteindre dans certains cas $0,5 \%$. Leur action serait donc nuisible à la quantité de beurre séerétée.

Les eoques de cacao, ainsi qu'il résulte d'expériences poursuivies à Gournay-sur-Marne, près de Paris, par M. J.-E. Lucas, avee l'aide des conseils du professeur Mallèvre, ont une influence nettement défavorable sur la production du lait et du beurre. La diminution a pu atteindre $20 \%$ de la quantité de lait et $20 \%$ de la quantité de beurre primitivement obtenues. L'influence nocive de ces coques varie d'ailleurs avec leur préparation antérieure; elle semble due, bien que cela ne soit pas explicitement démontré, à leur teneur en théobromine, alcaloïde spécifique dont l'action sur les sécrétions est bien connue.

$7^{\circ}$ Action des galactagogues. - Les galactagogues sont des substances qui, distribuées à faible dose, jouiraient du pouvoir d'intensifier la produetion mammaire. Il convient de citer, parmi ces produits, les feuilles de cotonnier, les feuilles d'ortie, de laurier blanc, la racine 
de tasi, plante de l'Amérique du Sud, et une petite plante de nos régions, le galega officinal. Utilisés en médecine humaine, les galactagogues ne semblent pas avoir gagné jusqu'à présent la confiance des éleveurs. Les expériences entreprises sur des vaches laitières, dans le but de démontrer leur efficacité, n'ont pas fourni de résultats positifs.

Dans l'alimentation des femmes qui allaitent, l'emploi de certaines de ces substances paraît cependant fournir d'heureux effets. Il y a sur ce point contradiction manifeste entre l'opinion du monde médical et celle des zootechniciens. Mais peut-être est-il possible de donner une explication de ces divergences de vue, car la physiologie générale nous interdit de faire appel à l'hypothèse d'un mécanisme différent du fonctionnement physiologique chez la femme, d'une part, et chez les femelles domestiques, d'autre part. L'être humain est éminemment sensible à la suggestion et le fait de disdistribuer à une patiente un galactagogue supposé accroît chez celle-ci la volonté d'avoir du lait. Or, cette simple action de la volonté peut très bien agir elle-même dans le sens d'un accroissement du fonctionnement mammaire, par l'influence du système nerveux. On conçoit sans peine que ce mécanisme soit sans action dans le cas de femelles animales, qui obéissent à l'instinct, mais non à la raison.

Mentionnons enfin, bien qu'il ne s'agisse pas à proprement parler d'une action alimentaire, l'effet galactagogue des injections de lait faites aseptiquement, par voie sous-cutanée (à la dose de 10 à 20 centimètres cubes).

Cette action parait fort nette, mais elle est de faible amplitude et sans grande importance au point de vue économique. Cependant, il convenait de la signaler, car la pratique des injections pourrait être considérée comme un "doping ", c'est-à-dire comme une fraude véritable, dans le cas où un éleveur malhonnête aurait l'intention de l'employer à l'occasion d'un concours beurrier. Les contrôleurs laitiers feront donc bien de s'assurer si les vaches destinées aux concours ne portent pas de trace de piqûres, ni d'abcès ou de grosseurs suspectes (1). Des injections sous-cutanées de corps nucléoprotéïques extraits de la mamelle, à la dose de 10 centimètres eubes, paraissent exercer également une action stimulante efficace, mais de très courte durée. (2)

(1) Cf. Recherches sur un nouveau galactogène, D. Brentana, Rivista di Agricoltura, Parme, 3 mai 1918.

(2) Recherches sur l'influence des nucléoprotéides de la mamelle dans la sécrétion lactée des vaches, R. Guulani, Clinica Veterinaria, Milan, 30 septembre 1918. 
$8^{\circ}$ Action des toxiques. - Nous ne pouvons passer sous silence, bien entendu, l'action des substances toxiques, qui nuisent, non seulement à la mamelle, mais à l'organisme tout entier. Nous nous bornerons toutefois à l'étude des empoisonnements les plus fréquents chez la vache laitière, en priant ceux de nos lecteurs qui voudraient étudier particulièrement cette question de vouloir bien se rapporter aux traités spéciaux sur la matière.

La consommátion de certaines herbes présentes dans les pâturages en plus ou moins grande quantité, peut occasionner des accidents graves.

Parmi ces plantes, qui renferment des produits toxiques apparqenant à la catégorie des alcaloïdes, il convient de citer les renoncules, la ciguë, la colchique d'automne. Les prèles, fréquents dans les prairies humides, sont également dangereux (1). Bien que, d'une manière générale, les animaux sachent éviter d'eux-mêmes ces végétaux, conduits par leur instinct, il convient néanmoins, pour éviter des empoisonnements toujours possibles, d'assainir les herbages, par des soins appropriés, tels que le draînage, le chaulage, la pratique du hersage et l'apport d'engrais. Nous conseillons de se méfier de la flore des fossés et d'éviter, autant que possible, de faire pacager les vaches laitières au voisinage de ces demiers, lorsque l'on s'est rendu compte de la présence de plantes suspectes dans la composition botanique de cette flore.

Les aliments concentrés sont parfois nuisibles. Le mélange accidentel de quelques graines de ricin aux arachides suffit pour rendre dangereux le tourteau qui résulte de la pression de ces graines. La semence du ricin contient en effet une toxine, de nature diastasique, qui agit à très petite dose et que l'on a comparé, en raison de ses propriétés biologiques, aux venins des serpents. Il y a peu de temps, en 1919, MM. Brioux, Directeur de la Station agronomique de Rouen, et Guerbet ont signalé une série d'accidents survenus en Seine-Inférieure, à la suite de la consommation de tourteaux qui contenaient de 1 à $2 \%$ de graines de ricin (2).

Le tourteau de coton non décortiqué renferme une substance

(1) Hoc, dans le Journal d'Agriculture pratique, a signalé récemment (1920) que du foin renfermant $33 \%$ de prèles, et distribué à des bovins, à la dose de 3 kilogrammes par jour, s'est montré toxique au point de faire périr 5 animaux sur 17.

(2) Cf. Ch. Brioux et M. Guereet, C. R. des séances de l'Académie d'Agriculture de France, 5 mai 1920. Les auteurs ont décrit dans ce travail une méthode biologique, basée sur les propriétés agglutinantes, vis-à-vis des globules sanguins, de la toxine du ricin. Cette méthode permet de retrouver jusqu'à $0,2 \%$ de tourteau de ricin mélangé à du tourteau d'arachide. 
toxique, le gossypol, qui appartient à la famille chimique des phénols ; il ne convient done pas aux vaches laitières. Le mélange de tourteau de coton non décortiqué au tourteau vendu comme décortiqué constitue une fraude dangereuse.

L'on sait aussi qu'un certain nombre de tourteaux, tels que ceux de crucifères de l'Inde, de mohra, de purghère, de noix d'arech, de croton, ne peuvent être donnés au bétail ; on doit les utiliser comme engrais.

Les fourrages envahis par les moisissures, ou qui ne paraissent pas dans un bon état de conservation, doivent être rejetés radicalelement de l'alimentation des vaches; c'est faire un mauvais caleul que de chercher à faire consommer à ces animaux des produits avariés, ne le seraient-ils que légèrement.

Les aliments fermentés, comme les drêches, les marcs de pomme, Tes pulpes, l'herbe ensilée, ne seront distribués qu'à faible dose, 15 kilogrammes par jour au maximum, et encore, sous la réserve expresse que ces produits exhalent bien une odeur franche et agréable; l'on évitera de fournir aux vaches des aliments ayant subi la fermentation butyrique, ce qui se reconnaît à la mauvaise odeur de la matière conservée.

Il est recommandé, si l'on utilise des pulpes de sucrerie - les pulpes de distillerie sont moins indiquées en raison de leur caractère acide - d'ensemencer ces pulpes avec des ferments lactiques (lactopulpe), avant de les mettre en silo (1).

Si l'on donne de l'ensilage de fourrages verts, l'on aura soin de s'assurer que la matière distribuée n'a pas été retirée du silo depuis plus de vingt-quatre heures. Le fourrage proviendra d'un silo établi en terrain sain, rempli avec de l'herbe verte, convenablement tassée et eonservée à l'abri de l'air humide.

Dans les locaux où l'on distribue des aliments fermentés, il faut veiller à la propreté scrupuleuse des mangeoires. Celles-ci seront nettoyées soigneusement après chaque repas; l'on évitera ainsi que des débris de matière ensilée restent presque indéfiniment devant les animaux et contaminent l'atmosphère de l'étable.

Les aliments mal conservés occasionnent chez les vaches des malaises, qui se traduisent souvent par des boiteries (dues à des lésions des articulations ou arthrites). Le lait qui provient de telles vaches peut être dangereux pour les enfants qui le consomment. II peut aussi provoquer des accidents chez les veaux et les porcelets, même s'il est distribué à ceux-ci sous forme de lait écrêmé.

(1) L'Institut Pasteur de Paris (Service des fermentations) délivre des ferments lactiques pour ensilage. Ce service est à la disposition des intéressés pour leur fournir tous renseignements utiles sur l'emploi de ces ferments. 
$9^{\circ}$ Influence des aliments sur la qualité du beurre. - Les propriétés physiques du beurre sont, dans une certaine mesure, sous la dépendance de l'alimentation. Certains fourrages donnent des beurres à point de fusion peu élevé, qui, en été, présentent vite un mauvais aspect sur le marehé. D'autres aliments, au contraire, possèdent la propriété d'augmenter le point de fusion, et par conséquent, de modifier favorablement la valeur commerciale du produit obtenu, pendant la période chaude de l'année. Voici comment les fourrages se classent à ce point de vue partieulier :

Aliments qui fournissent des beurres à point de fusion peu élevé

(de bonne qualité en hiver, trop mous en été)

Fourrages verts.

Tourteau de lin.

de colza.

de sésame.

de gluten de maïs,

Maïs en grain ou en farine.

Avoine en grain ou en farine.

Son de froment.
Aliments qui fournissent des beurres à point de fnsion élevé

(de bonne qualitéen été, trop durs en hiver)

Betteraves.

Feuilles et collets de betteraves.

Pommes de terre.

Tourteau de coprah.

- de palmiste.

de coton.

Légumineuses, en grain ou en farine. Son de seigle.

Les régimes alimentaires courants, à base de fourrages verts, en été, de betteraves, en hiver, ont tendance à donner des beurres dont la qualité commerciale n'est pas en rapport avec la saison. Il convient done de modifier les propriétés spécifiques de ces régimes par l'addition d'aliments concentrés appropriés. Ainsi, par exemple, il est recommandable de préférer pour l'été l'alimentation au tourteau de coprah. En hiver, si l'on ne veut pas avoir des beurres par trop durs, l'on choisira, comme aliments concentrés, le tourteau de lin et le son de froment.

L'on accuse certains aliments, eomme les choux-fourragers, le tourteau de colza, le tourteau de soja, l'herbe de certains pâturages, qui renferme des plantes ayant l'odeur de l'ail, de donner au lait une saveur désagréable. Autant que possible, l'on écartera ces produits de l'alimentation des vaches laitières. Mais, en ce qui concerne les ehoux-fourragers, il faut se garder de craindre outre mesure cet inconvénient. Si la ration de choux n'est pas trop importante, et si les mangeoires et les litières sont nettoyées périodiquement, de telle sorte que l'on puisse éviter la fermentation de débris végétaux à l'intérieur de l'étable, cette action désagréable du fourrage considéré n'est pas à craindre. Le lait prend plus facilement l'odeur de erucifère par son contact avec l'atmosphère du local, plutôt que par le passage direct du principe odorant à travers le corps de la vache. Nous avons consommé nous-même longtemps du beurre qui prove- 
venait de vaches nourries avec des choux-fourragers, mais entretenues dans des locaux très propres, et nous n'avons jamais observé la présence d'un goût spécial; ce beurre n'a d'ailleurs, jamais cessé d'avoir, au point de vue commercial, une excellente réputation.

\section{g) Influence du NOMBRE des repas, de LeUR COMPosition, DU MODE D'ABREUVEMENT.}

Les règles qui président à la distribution des aliments et des boissons sont assez élastiques. Ni le nombre, ni la composition des repas n'exercent une influence sensible sur la production laitière des bêtes. Ce qu'il importe avant tout de respecter, c'est la régularité dans les heures de distribution et dans l'ordre de succession des fourrages devant les vaches. Les animaux domestiques sont d'un naturel casanier; ils n'aiment pas ce qui ehoque leurs habitudes, et tout changement intempestif dans l'alimentation risque parfois de troubler certaines bêtes, et de diminuer momentanément dans une notable mesure la production de lait du troupeau tout entier.

Lorsque les animaux sont à l'étable, on leur donne habituellement deux repas par jour. En hiver, par exemple, l'on commencera par garnir les râteliers de foin, avant la traite du matin, puis le vacher se mettra à traire. Après cette opération, il sera procédé à la distribution des betteraves, hachées au coupe-racine et mélangées à la menue paille. L'on distribuera, pour terminer, les aliments concentrés.

A la suite d'un repos de quelques heures, le vacher recommencera, presque dans le même ordre, les mêmes opérations, immédiatement avant et après la traite du soir. Avant de quitter l'étable, il garnira le ratelier de paille d'avoine, afin de permettre aux bêtes fortes mangeuses de satisfaire leur appétit.

Nous donnons ce programme à titre purement indicatif. Peu importe, nous le répétons, l'ordre suivi dans la distribution. Mais lorsqu'un plan de rationnement a été adopté, l'essentiel est de s'y tenir, pour ne rien changer au régime des vaches. Autant que possible, il sera logique, cependant de distribuer d'abord les aliments qui plaisent le moins aux bêtes, pour terminer par ceux qui flattent davantage leur palais. Il appartient d'ailleurs au vacher de faire preuve d'esprit d'observation et d'associer les fourrages de la manière qui lui paraît la plus convenable.

Lorsque l'on doit passer d'un régime à un autre, il faut opérer d'une façon graduelle et ménager toute une série de paliers de transition. Si l'on veut introduire dans la ration un aliment nouveau, on le distribuera d'abord en petites doses, 100 à 200 grammes par 
tête, en le mélangeant, autant que possible, avec des fourrages appétissants, puis on augmentera peu à peu la quantité de ce produit. Les vaches refusent souvent les aliments qu'elles ne connaissent pas, et ce n'est que par des manœuvres patientes que l'on peut arriver à modifier leurs habitudes.

En ce qui concerne la distribution des boissons, nous avons dit précédemment que le meilleur régime était celui de l'alimentation ad libitum. Nous n'avons done pas besoin de revenir sur ce sujet.

\section{Pratique dlu contrồle de l'alimentation.}

\section{a) DÉtermination de la RAtion MOYENNe PAR tête.}

Pour apprécier la valeur pratique du rationnement d'un troupeau donné, il faut commencer par déterminer le poids moyen des vaches composant ce troupeau, soit avec l'aide d'une bascule, soit par un examen direct. L'on peut aussi utiliser un ruban métrique, à condition de se souvenir que les indications de ces rubans doivent être modifiées, d'après la conformation des sujets. Si l'on se sert, par exemple, du ruban de Crevat et si l'on mesure, avec ce ruban, des vaches laitières normandes, l'on prendra la précaution d'augmenter notablement le poids vif donné directement par la lecture de l'instrument. Afin d'apprécier l'importance de cette correction, le contrôleur aura soin, de peser d'abord et de mesurer immédiatement après, avec le ruban, un certain nombre de vaches; il profitera pour cela de son séjour sur une ferme dotée d'un pont-bascule, appareil qui permet de procéder commodément et rapidement aux pesées d'un grand nombre d'animaux.

Le calcul de la quantité moyenne de lait produit par tête et par jour est aisé; il consiste à diviser la quantité totale de lait récolté au cours des deux ou trois traites quotidiennes par le nombre de vaches présentes dans l'étable, sans se préoccuper si ces vaches sont en lactation ou dans leur période de repos.

Lorsque l'on connaît le poids d'une vache, d'une part, et la production moyenne par tête, d'autre part, il est facile de déterminer la valeur théorique, exprimée en unités fourragères, en protéines et en matière sèche, de la ration nécessaire, d'après les indications des paragraphes précédents.

Il reste à effectuer la reeherche de la ration moyenne par tête, réellement distribuée. L'on y parvient en entreprenant une enquête approfondie, au cours de laquelle le contrôleur doit faire preuve d'intelligence et d'initiative.

Supposons que nous nous trouvions en période d'alimentation hivernale. Les betteraves, la menue paille, le foin, constituent la 
partie volumineuse de la ration. Le complément est fourni aux animaux sous la forme d'aliments concentrés.

Il est facile de savoir, d'après le nombre de tombereaux conduits chaque jour du silo à la ferme, d'après la capacité du tombereau et la densité moyenne des betteraves conservées en tas (650 à 700 kilogrammes par mètre cube), le nombre de kilogrammes de betteraves mis à la disposition du vacher. L'on en déduit l'importance, à un ou deux kilogrammes près, de la ration individuelle de betteraves.

Si l'exploitation possède un pont-bascule, il est recommandable de peser le chargement réel d'un tombereau; cette détermination peut être faite une fois pour toutes.

Le contrôleur assistera ensuite à la préparation des betteraves; il se rendra compte, par le moyen de quelques pesées, effectuées avec la bascule romaine qui sert aux pesées de lait, de la proportion de balles de céréales rentrant dans la composition du mélange.

$\mathrm{Si}$, suivant les habitudes locales, l'on distribue le foin après l'avoir préalablement mis en bottes, il y aura lieu de déterminer le poids moyen d'une botte. L'on connaîtra, d'autre part, le nombre de bottes ou fractions de botte distribuées par tête, d'où l'on déduira le poids de la ration de foin.

Si le foin est distribué èn vrac, l'on déterminera le poids de la masse de foin placée devant chaque vache, en demandant au vacher de vouloir bien disposer, dans une partie inutilisée du râtelier de la vacherie, une masse comparable, par son volume, à la part servie à chaque animal pour un repas. Cette opération sera répétée deux ou trois fois, pour éviter les ehances d'erreurs, et l'on prendra la moyenne des résultats obtenus.

Les aliments concentrés sont généralement délivrés en sacs. Il est, par suite, extrêmement commode de se rendre compte de la quantité de ces aliments utilisée chaque jour; il suffit de peser les sacs avant, puis après les prélèvements opérés pour chaque repas. Le contrôleur ne doit pas seulement se fier, à ce point de vue, aux indications fournies par le fermier ou le vacher. Il doit se rendre compte par lui-même de la valeur des poids qu'il inscrira sur son carnet.

Le contrôle du rationnement, effectué comme nous venons de l'indiquer, sera fait à deux ou trois reprises dans le courant de l'hiver, à condition toutefois que la ration n'ait pas subi de modifications. Il conviendra de procéder à un nouveau contrôle chaque fois que l'on aura l'oceasion d'enregistrer un changement notable du programme alimentaire. Pendant la belle saison, lorsque les bêtes vont aux champs, l'on aura soin de noter l'étendue du pâturage réservé aux vaches, la valeur de ce pâturage, la durée du séjour quotidien 
des animaux dans les prés, Si les animaux changent de pâtures entre deux contrôles suceessifs, l'on s'efforcera d'obtenir des renseignements sur la date exacte de ces changements. Enfin, lorsque les vaches recevront un complément d'alimentation à l'étable, l'on déterminera aussi exactement que possible la composition de ce supplément, en employant la même méthode que pour le cas de l'alimentation d'hiver.

La connaissance de la composition de la ration moyenne va permettre d'établir la valeur fourragère des aliments réellement distribués, la comparaison de ces données - teneur en unités fourragères, teneur en protéines, teneur en matières sèches - avec les chiffres fournis par la considération de la ration théorique, fournira au contrôleur l'oecasion de vérifier si les vaches sont convenablement nourries. Le rapprochement de ces données est susceptible de fournir des indications pratiques très importantes.

Si, d'un contrôle à l'autre, l'on observe une chute brusque de la production laitière moyenne et s'il n'est pas possible d'expliquer cette chute par la mise au repos temporaire des vaches en fin de lactation, il y a lieu de suspecter l'insuffisance de la ration quotidienne. Ce soupçon se transformera en certitude, si, d'une part, il existe une différence notable (supérieure, par exemple, à une demiunité fourragère et à 100 grammes de protéines) entre la valeur fourragère de la ration théorique et la valeur correspondante des fourrages distribués et si, d'autre part, l'on constate une diminution du poids vif moyen, accompagné d'un changement de l'état général des vaches. Les bêtes insuffisamment nourries se reconnaissent facilement à leurs hanches saillantes, leur flanc creusé, leur peau légèrement adhérente au corps. Les indications de la bascule, ou, faute de mieux, celles que peut fournir le ruban métrique, viennent utilement compléter en la circonstance, les résultats de l'observation pure et simple, et facilitent le diagnostic.

Il convient de noter que le déficit alimentaire produit des effets d'autant plus nets que les bêtes sont moins avancées en lactation; e'est donc en observant les meilleures laitières que l'on se rendra le mieux compte de l'efficacité d'une alimentation déterminée.

Au contraire, si le contrôleur constate une légère augmentation du poids vif moyen, sans modification sensible de la production laitière, il doit, selon les circonstances, choisir entre deux conclusions différentes.

Lorsque le contrôle de l'alimentation fournit l'indication d'une ration théorique à peu près équivalente à la ration effective, il n'y a pas lieu de modifier le programme de rationnement, l'on peut ètre sûr que les animaux sont nourris dans d'excellentes conditions. 
Par contre, si le gain moyen de poids coexiste avec une ration dont la valeur nutritive dépasse notablement les indications théoriques, l'on se trouve dans un cas très net d'alimentation pléthorique, et il est possible de réaliser des économies sensibles, sans nuire à la production laitière, au moyen de la diminution des fourrages utilisés.

Le contrôleur laitier a le devoir de mettre immédiatement les propriétaires d'animaux au courant de ses observations. Il doit s'efforcer de jouer auprès de ces derniers le rôle de conseiller technique en matière de rationnement, car ses fonctions lui permettent de juger, mieux que personne, de la valeur des programmes d'alimentation suivis dans les fermes qu'il visite.

Mais le travail du contrôleur ne se borne pas à cette constatation; celui-ci doit encore s'assurer que la répartition de l'ensemble des aliments entre toutes les vaches s'effectue d'une façon rationnelle.

Parmi les bêtes contrôlées, les unes, prêtes à vêler, sont taries depuis quelque temps; les autres, qui viennent de mettre bas, fournissent une quantité journalière de lait dont l'ordre de grandeur est d'une vingtaine de litres. La production de leurs compagnes varie entre ces deux extrêmes et très peu de celles-ci se trouvent, en définitive, dans les conditions indiquées par les chiffres moyens du contrôle laitier. Bien entendu, les bêtes qui donnent moins de lait recevront les rations les moins fortes, tandis que les meilleures laitières seront nourries plus abondamment. Il appartient au vacher d'assurer à ehaque vache la quantité de nourriture qui lui est nécessaire, en modifiant notamment les rations d'aliments concentrés. Les tourteaux, par exemple, devront disparaitre de l'alimentation des bêtes taries, tandis que les vaches en pleine période de lactation recevront une quantité de ces aliments double de la dose moyenne. Le contrôleur se bornera à surveiller ce travail, sans intervenir le moins du monde dans la répartition, et il fera part ultérieurement au chef d'exploitation du fruit de ses observations.

La représentation graphique des résultats du contrôle laitier permet d'ailleurs à un observateur attentif de constater l'influence de la ration sur la production des vaches. Le document de la page suivante choisi à dessein, montre elairement l'utilité pratique de semblables constatations.

Dans l'exploitation $\mathrm{n}^{0} 2$, contrôlée par le service de Seine-etOise, la vache $n^{0} 3$, de race hollandaise, a vêlé le 4 janvier dernier,

Contrôlée peu de temps après son vêlage, elle donnait 19 kilogrammes de lait, contenant 600 grammes de matière grasse. Son poids était à ce moment de 550 kilogrammes.

Trois semaines après, nouveau contrôle. Les productions laitière 


\section{Exploitation de $M P$ - $\quad \ldots \quad n \cdot 2$ \\ FICHE DE CONTROLE LAITIER \& BEURRIER}

\section{Race deoleandarile}

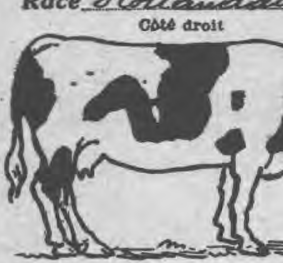

Achetée

Vendro

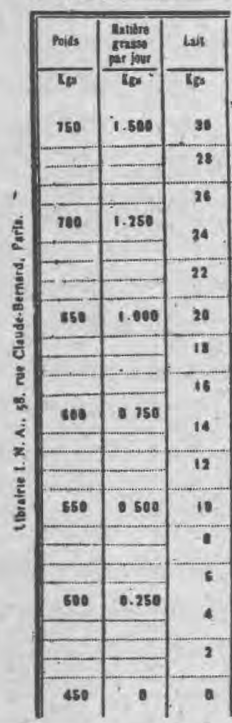

\section{Annee 1923}

Annee $1923 \quad N^{\circ} \mathcal{2}$

$N^{\circ} \mathcal{z}$ cote gauche

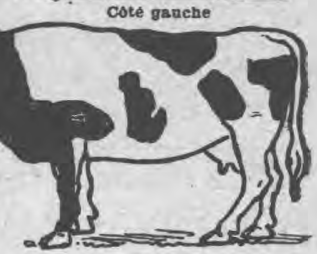

Berd Book

Ague de to and

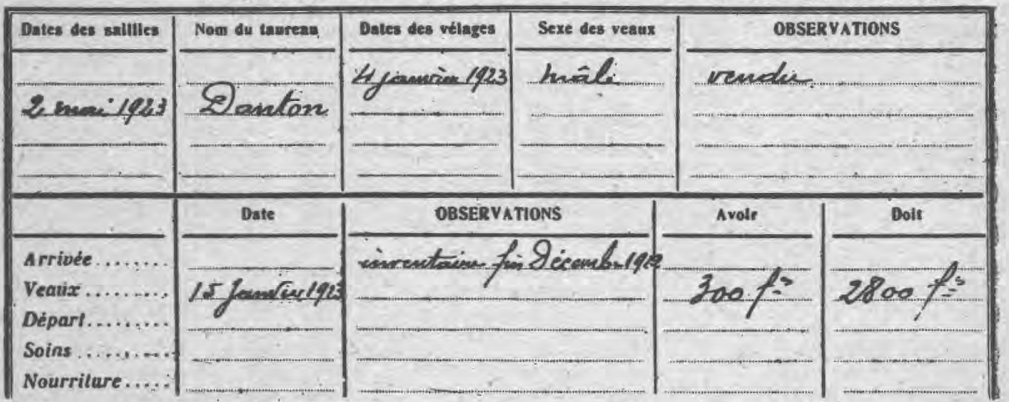


et beurrière avaient considérablement augmenté, tandis que le poids s'était abaissé à 500 kilogrammes.

Vers la fin de février, le contrôleur constate une baisse très accentuée du lait et de la matière grasse aceompagnée d'un nouvel affais-

- sement du poids. Une brève enquête lui démontre que la vache, insuffisamment nourrie, est obligée de prendre sur ses réserves de graisse pour faire face aux besoins de sa production; de suite, cet état de chose est signalé au chef de l'exploitation, qui donne immédiatement l'ordre d'augmenter d'un kilogramme de tourteau la quantité des fourrages distribués à la vache en question. Celle-ci réagit immédiatement à l'amélioration de son régime. Un mois après, la quantité de lait a augmentée de 2 litres et le poids est repassé de 490 à 520 kilogrammes.

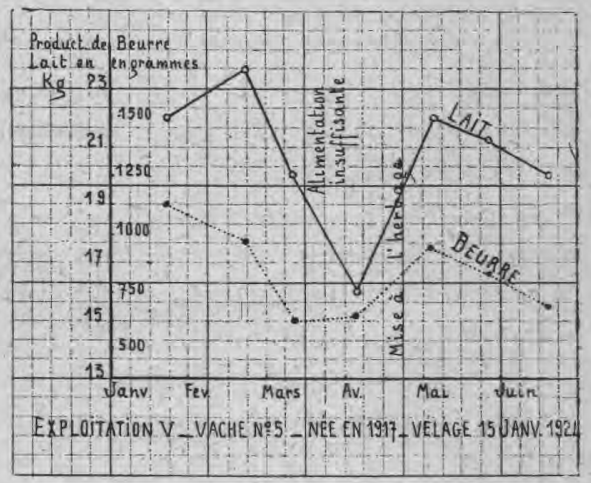

Dans une autre exploitation, une bête qui donnait $23 \mathrm{~kg}$. 7 de lait au moment de son vêlage, en février, se trouvait insuffisamment alimentée, malgré les observations faites au vacher. La chute de la courbe de lactation s'est alors montrée très rapide; cependant, quelques mois après, au moment de la mise à l'herbe, la production quotidienne est brusquement remontée, d'un contrôle à l'autre, de 6 kilogrammes. Une pareille constatation fournit la preuve manifeste de l'erreur commise antérieurement Soumise à un régime plus favorable, cette vache aurait certainement donné, de fin février à mai, e'est-à-dire pendant soixante-dix jours, un excédent de production quotidienne d'au moins 3 kilogrammes. Avec un meilleur mode de nourriture, la lactation totale de cette vache aurait done été augmenté de 210 kilogrammes, ce qui est loin d'ètre négligeable.

Avec un.peu d'habitude, la lecture des graphiques de contrôle s'effectue avec une grande rapidité. Les contrôleurs doivent s'en- 
traîner à ce travail, qui leur permet de donne d'utiles conseils, aussi bien aux vachers qu'aux chefs des exploitations visitées. Quelques exploitants ont pris l'habitude de distribuer une prime à leurs vachers, ealculée, non pas suivant la production laitière totale de chaque jour, ce qui peut parfois conduire à la fraude par mouillage, avec un personnel malhonnête, mais d'après les résultats moyens constatés par le contrôleur laitier le jour de sa visite. Le contrôleur peut être sûr, dans ce cas, que les indications qu'il donne en matière d'alimentation seront suivies, car il est de l'intérêt du vacher de nourrir convenablement les bonnes bêtes, afin de les maintenir toujours dans le meilleur état possible de lactation.

C'est surtout à la fin de l'hiver et à la fin de l'été qu'il convient de veiller au rationnement des vaches. Ces époques constituent, à n'en pas douter, deux périodes essentiellement eritiques au point de vue de l'alimentation des bêtes laitières. Il est facile d'en indiquer les raisons.

En février-mars, les provisions de l'hiver s'épuisent, et l'on a tout naturellement tendance à procéder aux distributions d'aliments avec parcimonie. D'autre part, la conservation des betteraves, principal constituant de nos régimes d'hiver, ne va pas sans une certaine perte de substance, sur laquelle on ne saurait jamais trop attirer l'attention des praticiens. Il découle de cette perte que la valeur fourragère des betteraves, au moment de l'arrachage, est supérieure à celles des racines conservées pendant plusieurs mois, si bonnes que soient les conditions de cette conservation. Une ration qui, au début de l'hiver, se trouve équilibrée avec 40 à 50 kilogrammes de betteraves, cesse de l'être avec le même poids des racines d'arrièresaison, et, comme l'on ne se méfie pas de cette diminution de valeur nutritive, il en résulte que le régime alimentaire des bêtes reste défectueux jusqu'aux approches du printemps. Nous en voyons la preuve dans l'importance de l'augmentation de production provoquée par la mise à l'herbage.

Dans une ferme que nous connaissons, et où l'on nourrit fortement, eette augmentation est de $1 \mathrm{~kg}$. 5 , en moyenne, par tếte; elle est de 3 kilogrammes, au contraire, dans un autre domaine, dont le propriétaire n'apporte pas la même attention à la eonduite de son troupeau; les deux graphiques de production laitière, que nous reproduisons ici sont empruntés aux fiches des troupeaux considérés, ils accusent très clairement la valeur pratique de ce déficit, auquel il est si facile de remédier, lorsqu'on s'en aperçoit, par la distribution d'une quantité d'aliments concentrés supplémentaires.

Vers le mois d'août, par contre, e'est le régime de la prairie qui risque de se montrer déficitaire. Lorsque la densité du troupeau n'est 
pas proportionnée à la richesse de l'herbage et lorsqu'une période de sécheresse prolongée entrave la pousse de l'herbe, les bêtes souffrent d'une inanition partielle, et leur production laitière diminue plus rapidement qu'elle ne devrait. L'on peut enrayer facilement cette baisse par la distribution, au besoin sur la prairie même, d'une

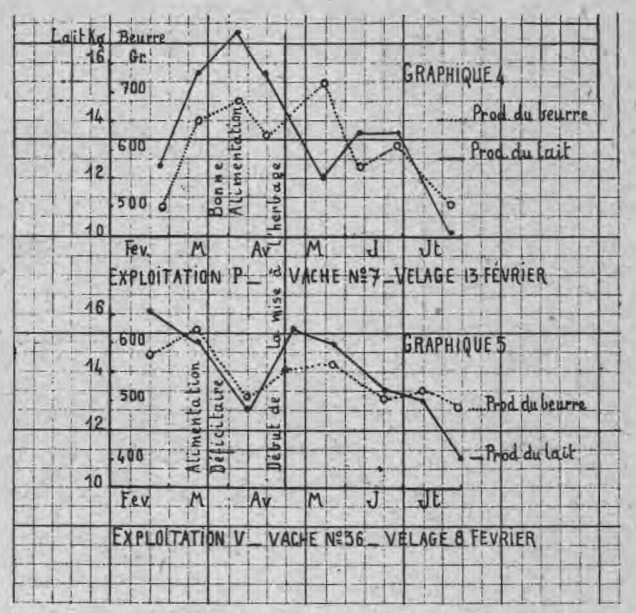

Ces deux vaches ont vêlé à la même époque.

L'une (le $\mathrm{n}^{\circ} 7$ ) a été bien nourrie; elle donne son maximum de lait un mois et demi après vêlage; la mise à l'herbe ne fait remonter sa production de lait que de $1 \mathrm{~kg}$. 5 .

L'autre (le $n^{\circ} 36$ ) a été insuffisamment nourrie, pendant la période critique de février-mars; elle baisse de lait aussitôt après son vêlage; de plus, la mise à l'herbe fait remonter sa production de 3 kilogrammes.

certaine quantité de fourrage de luzerne, coupé vert, ou encore, ce qui est préférable pour lés très fortes laitières à capacité digestive restreinte, par une dose correspondante de tourteau d'arachide ou de tourteau de lin (1).

Le graphique suivant montre l'influence favorable de la distribution de regain de luzerne, au cours d'une période de sécheresse; la chute rapide de la courbe avant cette distribution trahissait, pour un observateur exercé, l'insuffisance alimentaire du pâturage. C'est à la suite de l'observation d'un contrôleur laitier que cette distribution a été ordonnée et exécutée; on en constate facilement les heureux effets. Toutes les courbes des meilleures laitières de l'étable

(1) Il convient alors de faire rentrer les vaches à l'étable, car la distribution des tourteaux au pâturage n'est guère pratique. 
considérée ont d'ailleurs réagi de la mềme manière, avec ún parallélisme frappant.

Ajoutons, pour terminer, que le passage d'une alimentation normale à un régime de sous-nutrition produit des effets immédiats; un ou deux jours après le changement, la production laitière s'abaisse

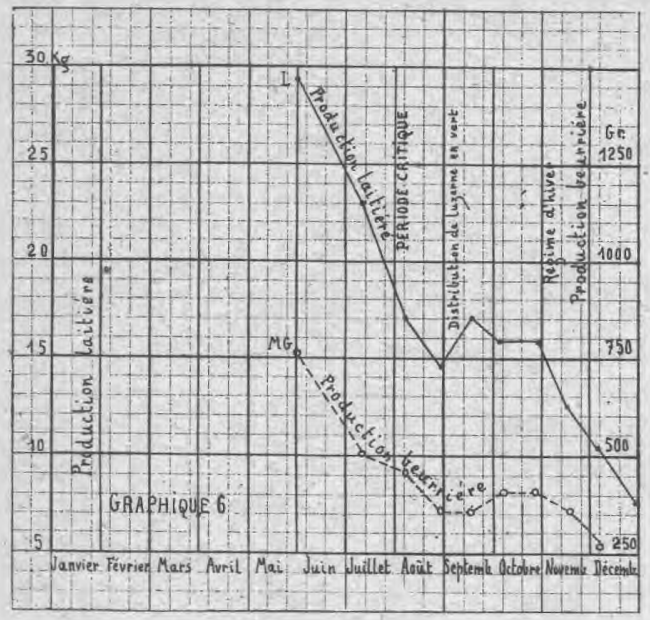

La vache dont voici la courbe de lactation a vêlé le 5 mai rg23. En raison de sa grande aptitude laitière, le régime du pâturage exclusif n'a pas tardé à ètre insuffisant pour elle. Cetle insuffisance s'est particulièrement accentuée en août. Le 15 août, l'on a procédé à une distribution supplémentaire de regain de luzerne. On en constate facilement les eflets, grâce aux résultats des contrôles de septembre et d'octobre.

nettement, tandis que le taux butyreux s'accroît. Cependant, cet accroissement du pourcentage de matière grasse est insuffisant pour compenser la diminution de quantité du lait sécrété ; il s'en suit que la production du beurre se trouve également modifiée dans un sens défavorable par l'effet du changement de ration.

Le graphique ci-joint, que nous empruntons à un récent travail américain (1), traduit encore mieux que ne le feraient des chiffres le phénomène envisagé. La mamelle réagit done à un abaissement de valeur nutritive de la ration par un abaissement de la sécrétion du lait et du beurre, accompagnée d'une élévation du taux butyreux. Cette observation mérite d'être retenue, car elle peut permettre à un contrôleur habile de déceler en temps opportun une erreur d'alimentation, qui consisterait par exemple dans la substitution d'un

(1) Arthur-C. Ragsdale et Ch.- W. Turner, Effects of underfeeding on milk secretions (Journal of Dairy Science, vol. VI, $\mathrm{n}^{\circ} 4$, juillet 1923). 
aliment beaucoup plus pauvre que ne l'indiquent les tables à un fourrage de bonne qualité, dont la valeur fourragère n'était pas appréciée à sa véritable valeur. De telles erreurs ne sont pas impossibles; car, ne l'oublions pas, nos tables ne fournissent que des prévisions forcément approximatives, et il convient de corriger dans le sens indiqué par l'observation pratique les indications que l'on peut en tirer.

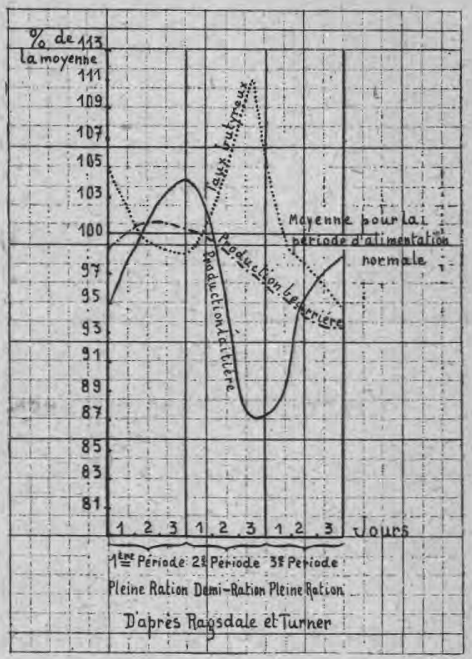

L'étude des courbes de contrôle, qui permet l'appréciation de la valeur effective d'un régime déterminé, facilite cette adaptation des tables aux conditions de la pratique, et l'on ne saurait trop attirer l'attention des contrôleurs laitiers sur l'importance de cette question.

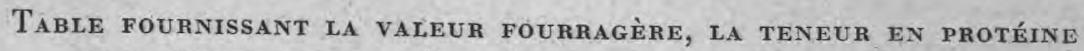
ET LA TENEUR EN MATIÈRE SÈCHE DES PRINCIPAUX ALIMENTS DE LA VACHE LAITIÈRE.

1 . Fourrages verts.

\begin{tabular}{|c|c|c|c|c|c|}
\hline \multirow{3}{*}{ Nature du Pourrage } & \multirow{2}{*}{$\begin{array}{c}\text { Valeur de l'équivalent } \\
\text { représentant } \\
\text { une unité en } \mathrm{kg} \text {. }\end{array}$} & \multicolumn{3}{|c|}{$\begin{array}{c}\text { Protếnes } \\
\text { en gr. par kg. de P'aliment } \\
\end{array}$} & \multirow{2}{*}{$\begin{array}{l}\text { Matière sèche } \\
\text { en kg. par Iòo kg. } \\
\text { do l'aliment }\end{array}$} \\
\hline & & $\begin{array}{l}\text { de nature } \\
\text { albuminoide } \\
-\end{array}$ & $\begin{array}{l}\text { de nature } \\
\text { non albuminoiide }\end{array}$ & $\overline{\text { Totales }}$ & \\
\hline & $\begin{array}{l}(\bar{a}) \\
\text { kiogr. }\end{array}$ & $\begin{array}{l}\overline{(b)} \\
\text { gr. }\end{array}$ & $\begin{array}{l}\text { (amides) } \\
(c) \\
\text { g:. }\end{array}$ & $\begin{array}{l}\overline{(a)} \\
\text { gr. }\end{array}$ & $\begin{array}{c}\overline{(e)} \\
\text { kilogx. }\end{array}$ \\
\hline moyenne. & 6,5 & 17. & 8 & 25 & 20 \\
\hline ure.. & 5,5 & 23 & 11 & 34 & 22 \\
\hline rrage . & 6,5 & 14 & 7 & 21 & 23 \\
\hline
\end{tabular}




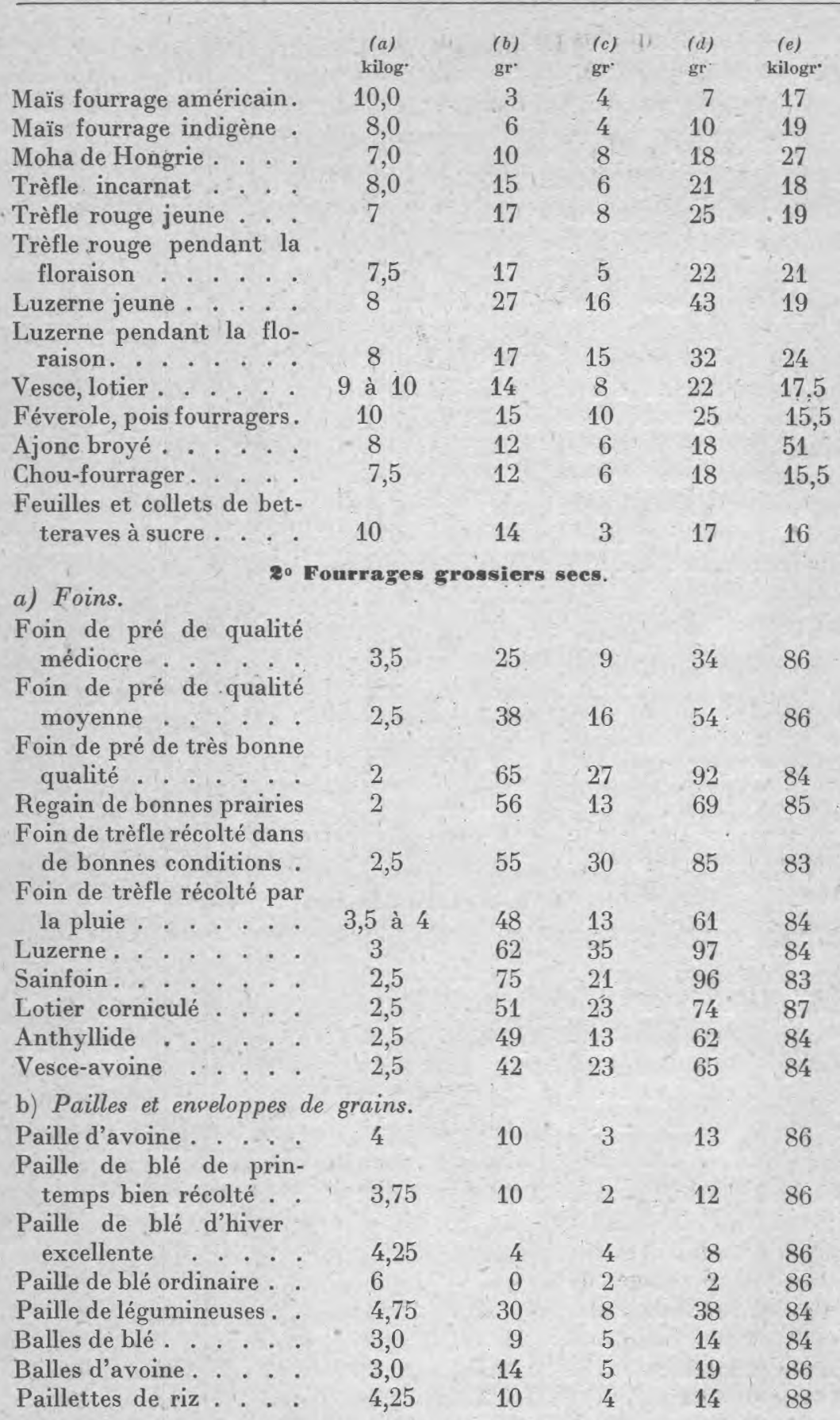


3. Fourrages verts ensilés.

\begin{tabular}{|c|c|c|c|c|c|}
\hline & $\begin{array}{c}(a) \\
\text { kilogr. }\end{array}$ & $\begin{array}{l}\text { (b) } \\
\text { gr. }\end{array}$ & $\begin{array}{l}\text { (c) } \\
\text { gr. }\end{array}$ & $\begin{array}{l}\text { (d) } \\
\text { gr. }\end{array}$ & $\begin{array}{c}\text { (e) } \\
\text { kilogr. }\end{array}$ \\
\hline $\begin{array}{l}\text { Ensilage d'herbe de prai- } \\
\text { rie . }\end{array}$ & 9 & 9 & 5 & 14 & 19 \\
\hline Ensilage de maïs . . . . & 8 & 4 & 4 & 8 & 18 \\
\hline $\begin{array}{l}\text { Ensilage de luzerne. } \\
\text { Ensilage de feuilles et de } \\
\text { collets de betteraves }\end{array}$ & 10 à 11 & 15 & 10 & 25 & 17 \\
\hline sucrières . . . . . & 7,5 & 2 & 13 & 15 & 23 \\
\hline
\end{tabular}

\section{Racines et tubercules.}

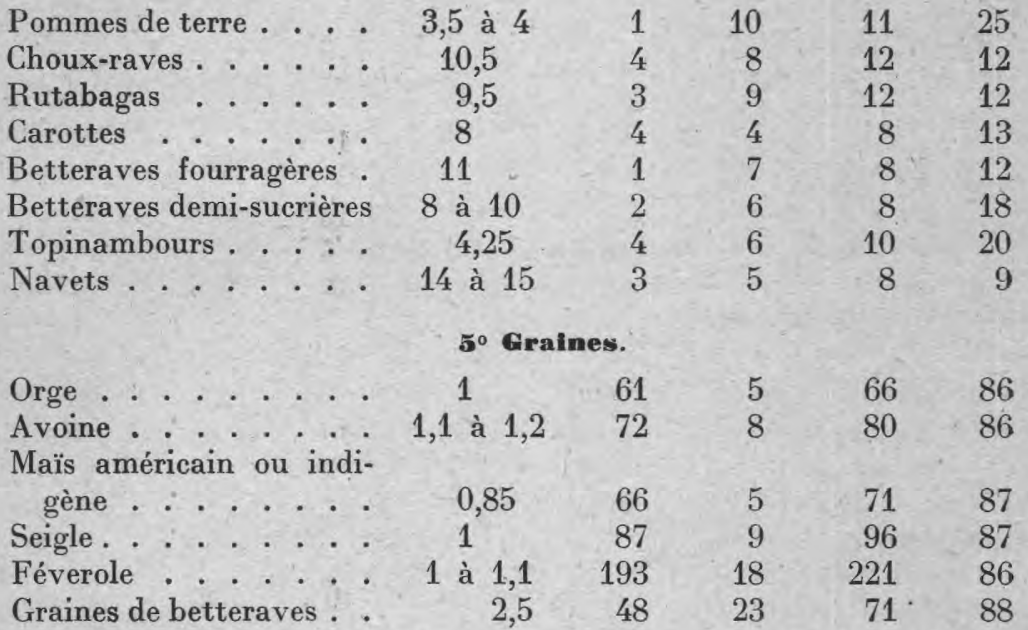

Résidus industriels.

a) Résidus de meunerie.

Gros son de froment. . Recoupes ...... Farines basses de riz... Déchets de polissage de riz . . . . . . Gluten de maïs . . . .

b) Résidus de sucrerie.

$\begin{array}{rrrrr}1,6 & 98 & 15 & 113 & 88 \\ 1,0 & 110 & 10 & 120 & 88 \\ 1 & 60 & 8 & 68 & 87\end{array}$

$\begin{array}{rrrrr}1,2 & \prime \prime & \prime \prime & n & \prime \prime \\ 1,1 & 184 & 15 & 199 & 92\end{array}$

Pulpes de betteraves à la sortie des diffuseurs.

Pulpes de betteraves à la sortie des presses ....

Pulpes de betteraves conservées par ensilage. . Pulpes desséchées . .

$\begin{array}{rrrr}3 & 2 & 5 & 12 \\ 36 & 5 & 41 & 89 \\ 0 & 54 & 54 & 78\end{array}$


c) Résidus de brasserie, de distillerie et de cidrerie.

Drêches de brasserie fraîches . . . . .

Drêches de brasserie desséchées . . . 1,2 à 1,4 137

Touraillons . . . $1,8 \quad 114$

Marc de pommes frais..

Marc de pommes sec..

Marc de raisin, avec râfles

(a)

kilogr.

$$
5 \text { à } 6
$$

7,5

2,5

28

d) Résidus d'huilerie.

Tourteau de coton décortiqué ........

Tourteau d'arachide Rufisque ......

Tourteau d'arachide ordinaire .......

Tourteau de coprah.

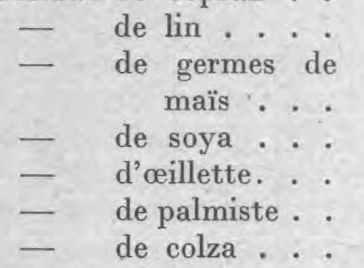

(b) gr.

(c)

(d)

(a)

kilogr.

2

37

24

20

$114 \quad 71$

3

12

3

$\begin{array}{rrr}9 & 146 & 88 \\ 71 & 185 & 88 \\ 1 & 4 & 20 \\ 4 & 16 & 90 \\ 2 & -5 & 30\end{array}$

$\begin{array}{lrrrr}0,95 & 407 & 16 & 423 & 91 \\ 0,90 & 452 & 15 & 467 & 91 \\ 0,95 & 387 & 13 & 400 & 90 \\ 0,90 & 163 & 4 & 167 & 90 \\ 0,95 & 272 & 16 & 288 & 89 \\ 0,90 & 144 & 39 & 183 & 89 \\ 0,90 & 399 & 8 & 407 & 89 \\ 1,00 & 266 & 16 & 282 & 89 \\ 0,90 & 146 & 4 & 150 & 90 \\ 1,1 & 230 & 44 & 274 & 90\end{array}$

e) Résidus de laiterie et divers.

Lait écrémé . . . . . 8

Babeurre . . . . . 8

Petit lait de fromagerie.

Sang desséché . . . .

Farine de poisson . .

Farine de viande. . . .

11 à 12

1

1,6

0,750
38

38

9

680

436

636

\begin{tabular}{ccr}
0 & 38 & 10 \\
0 & 38 & 10 \\
0 & 9 & 7 \\
92 & 772 & 91 \\
37 & 473 & 88 \\
36 & 672 & 90 \\
\multicolumn{3}{c}{ (A suivre). }
\end{tabular}

\section{LE PROCÈS DE LA MATIĖRE GRASSE DU LAIT} par Ch. PORCHER

- suite -

Les variations butyreuses du lait chez la femme. Lorsqu'il s'agit d'étudier, au point de vue qui nous occupe en ce moment, la sécrétion lactée de la femme, une difficulté se présente, que nous n'avons pas jusqu'ici rencontrée : c'est l'impossibilité, dans l'espèce humaine, d'avoir un échantillon réellement moyen.

Chez toutes les femelles domestiques orientées vers la production industrielle du lait : vache, chèvre, brebis, il est facile d'effectuer 